\title{
Rapid engraftment of human ALL in NOD/SCID mice involves deficient apoptosis signaling
}

\author{
M Queudeville ${ }^{1,2}$, F Seyfried ${ }^{1,2}$, SM Eckhoff ${ }^{1}$, L Trentin ${ }^{1}$, S Ulrich ${ }^{1}$, M Schirmer ${ }^{1}$, K-M Debatin ${ }^{\star, 1}$ and LH Meyer ${ }^{\star, 1}$
}

Previously, we found that rapid leukemia engraftment (short time to leukemia, TTL ${ }^{\text {short}}$ ) in the NOD/SCID/huALL (non-obese diabetic/severe combined immuno-deficiency/human acute lymphoblastic leukemia) xenograft model is indicative of early patient relapse. As earlier intact apoptosis sensitivity was predictive for good prognosis in patients, we investigated the importance of apoptosis signaling on NOD/SCID/huALL engraftment. Intact apoptosome function as reflected by cytochrome c-related activation of caspase-3 (CRAC-positivity) was strongly associated with prolonged NOD/SCID engraftment (long time to leukemia, $T T^{\text {long }}$ ) of primary leukemia cells, good treatment response and superior patient survival. Conversely, deficient apoptosome function (CRAC-negativity) was associated with rapid engraftment (TTL ${ }^{\text {short }}$ ) and early relapse. Moreover, an intact apoptosis signaling was associated with high transcript and protein levels of the pro-apoptotic death-associated protein kinase 1 (DAPK1). Our data strongly emphasize the impact of intrinsic apoptosis sensitivity of ALL cells on the engraftment phenotype in the NOD/SCID/huALL model, and most importantly also on patient outcome.

Cell Death and Disease (2012) 3, e364; doi:10.1038/cddis.2012.107; published online 9 August 2012

Subject Category: Experimental Medicine

Despite major achievements in the treatment optimization of pediatric patients with acute lymphoblastic leukemia (ALL) that have led to increased cure rates up to more than $80 \%$ over the last decades, relapse of the disease is associated with poor prognosis, ${ }^{1,2}$ especially if occurring at early time points. ${ }^{3}$ Upfront identification of relapse patients based on prognostic markers including molecular minimal residual disease is hampered by the fact that the majority of patients encountering relapse are not recognized by these markers and are initially assigned to non-high-risk therapy regimens. ${ }^{2,4}$ This highlights the need to identify prognostic markers, which ideally also point to novel therapeutic approaches for (early) relapse patients.

In a recent study we have characterized engraftment of patient ALL samples transplanted onto NOD/SCID (nonobese diabetic/severe combined immuno-deficiency) mice and identified that a short time between transplantation and onset of leukemia-related morbidity in the recipient animals (short time to leukemia, TTL ${ }^{\text {short}}$ ) is a strong prognostic factor indicative for early relapse independently of established markers. The TTL ${ }^{\text {short }}$ phenotype was further characterized by a gene expression signature pointing to altered survival/cell death (cd) pathways in high risk/early-relapse leukemia. ${ }^{5}$ Deregulated apoptosis or programmed cd is considered to be an important feature of malignant transformation ${ }^{6}$ and has been implicated in resistance and the treatment failure of acute leukemia. ${ }^{7,8}$ To address whether TTL ${ }^{\text {short/early-relapse }}$ leukemia is characterized by functionally relevant altered apoptosis signaling, we analyzed apoptosome function in patient derived ALL xenografts and evaluated its impact on NOD/SCID/huALL engraftment, treatment response and overall patient survival.

\section{Results}

Xenograft ALL and TTL. Twenty-three leukemia xenografts which were established employing our NOD/SCID/huALL xenotransplant mouse model were investigated in this study. All xenografts were derived from de novo diagnosed pediatric B-cell precursor ALL patients (leukemia and patient characteristics are summarized in Table 1). After transplantation, recipient animals were regularly examined for the onset of leukemia and killed upon disease manifestation. The presence of high leukemia infiltration was confirmed in

\footnotetext{
${ }^{1}$ Ulm University, University Medical Center Ulm, Department of Pediatrics and Adolescent Medicine, Eythstrasse 24, 89075 Ulm, Germany

${ }^{*}$ Corresponding author: K-M Debatin or LH Meyer, Ulm University, University Medical Center Ulm, Department of Pediatrics and Adolescent Medicine, Eythstrasse 24, 89075 Ulm, Germany. Tel: +49 731500 57254/-57001; Fax: +49 731500 57002; E-mail: klaus-michael.debatin@uniklinik-ulm.de (K-MD) or lueder-hinrich.meyer@uniklinik-ulm.de (LHM).

${ }^{2}$ These authors contributed equally to this work.

Keywords: acute lymphoblastic leukemia; apoptosis signaling; xenograft model; NOD/SCID; prognosis

Abbreviations: ALL, acute lymphoblastic leukemia; ABL, abelson oncogene; ac, activated caspase-3; AML, acute myeloid leukemia; AMP, adenosine monophosphate; B2M, beta-2-microglobulin; Bax, Bcl-2-associated X protein; Bcl-2, B-cell lymphoma-2; BCP, B-cell precursor; BCR, breakpoint cluster region; BFM, Berlin Frankfurt Münster study group; c-ALL, common acute lymphoblastic leukemia; cc, cytochrome c; cd, cell death; CRAC, cytochrome c-related activation of caspase-3; DAPK1, death-associated protein kinase1; ETV6, ETS (E-twenty six) variant 6; FITC, fluorescein isothiocyanate; HR, high risk; Mcl-1, myeloid cell leukemia sequence 1; MLL, mixed lineage leukemia; NOD, non-obese diabetic; PAGE, polyacrylamide gel electrophoresis; PCR, polymerase chain reaction; PDE4A, phosphodiesterase 4A; PE, phycoerythrine; PR, prednisone response; RPMI, Roswell Park Memorial Institute; RUNX1, runt-related transcription factor 1; SCID, severe combined immuno-deficiency; SDHA, succinate dehydrogenase A; SDS, sodium dodecyl sulfate; SEM, standard error of the mean; TBP, TATA box-binding protein; TTL, time to leukemia; XIAP, X-linked inhibitor of apoptosis

Received 3.4.12; revised 25.6.12; accepted 5.7.12; Edited by $Y$ Shi
} 
Table 1 Characteristics of ALL samples and derived xenografts

\begin{tabular}{|c|c|c|}
\hline & $N$ & $\%$ \\
\hline Total & 23 & 100 \\
\hline \multicolumn{3}{|l|}{$\begin{array}{l}\text { Patient characteristics } \\
\text { Gender }\end{array}$} \\
\hline $\begin{array}{l}\text { Female } \\
\text { Male }\end{array}$ & $\begin{array}{l}10 \\
13\end{array}$ & $\begin{array}{l}43 \\
57\end{array}$ \\
\hline Age & & \\
\hline $\begin{array}{l}1-9 \text { years } \\
0-1 \text { and }>9 \text { years }\end{array}$ & $\begin{array}{r}15 \\
8\end{array}$ & $\begin{array}{l}65 \\
35\end{array}$ \\
\hline $\begin{array}{l}\text { Immunophenotype } \\
\text { pro-B ALL } \\
\text { C-ALL } \\
\text { pre-B ALL }\end{array}$ & $\begin{array}{r}2 \\
14 \\
7\end{array}$ & $\begin{array}{r}9 \\
61 \\
30\end{array}$ \\
\hline $\begin{array}{l}\text { Fusion gene } \\
\text { ETV6-RUNX1 } \\
\text { BCR/ABL } \\
\text { MLL rearrangement } \\
\text { No }\end{array}$ & $\begin{array}{r}4 \\
1 \\
2 \\
16\end{array}$ & $\begin{array}{r}17 \\
4 \\
9 \\
70\end{array}$ \\
\hline $\begin{array}{l}\text { Prednisone response } \\
\text { Good } \\
\text { Poor }\end{array}$ & $\begin{array}{r}22 \\
1\end{array}$ & $\begin{array}{r}96 \\
4\end{array}$ \\
\hline $\begin{array}{l}\text { Day } 15 \text {, blast cell persi } \\
>5 \% \\
<5 \% \\
\text { Not available }\end{array}$ & $\begin{array}{l}7 \\
7 \\
9\end{array}$ & $\begin{array}{l}30 \\
30 \\
40\end{array}$ \\
\hline $\begin{array}{l}\text { BFM risk groups } \\
\text { Non-HR } \\
\text { HR }\end{array}$ & $\begin{array}{r}19 \\
4\end{array}$ & $\begin{array}{l}83 \\
17\end{array}$ \\
\hline $\begin{array}{l}\text { Relapse }^{a} \\
\text { Yes } \\
\text { No }\end{array}$ & $\begin{array}{r}5 \\
18\end{array}$ & $\begin{array}{l}22 \\
78\end{array}$ \\
\hline $\begin{array}{c}\text { Xenograft characteristics } \\
\text { Time to leukemia (TTL }\end{array}$ & & \\
\hline $\begin{array}{l}\text { Long } \\
\text { Short }\end{array}$ & $\begin{array}{r}16 \\
7\end{array}$ & $\begin{array}{l}70 \\
30\end{array}$ \\
\hline $\begin{array}{l}\text { CRAC } \\
\text { Positive } \\
\text { Negative }\end{array}$ & $\begin{array}{r}16 \\
7\end{array}$ & $\begin{array}{l}70 \\
30\end{array}$ \\
\hline $\begin{array}{l}\text { NOD/SCID passage us } \\
\text { P } 0 \\
\text { P } 1 \\
\text { P } 2 \\
\text { P } 3 \\
\text { P } 4 \\
\text { P } 5 \\
\text { P } 6\end{array}$ & $\begin{array}{c}\text { alysis } \\
1 \\
8 \\
6 \\
4 \\
2 \\
1 \\
1\end{array}$ & $\begin{array}{r}4 \\
36 \\
26 \\
17 \\
9 \\
4 \\
4\end{array}$ \\
\hline
\end{tabular}

Abbreviations: $A B L$, abelson oncogene; $A L L$, acute lymphoblastic leukemia; $\mathrm{BCR}$, breakpoint cluster region; BFM, Berlin Frankfurt Münster study group; CRAC, cytochrome c-related activation of caspase-3; ETV6, ETS (E-twenty six) variant 6; MLL, mixed lineage leukemia; NOD, non-obese diabetic; RUNX1, runt-related transcription factor 1 ; SCID, severe combined immuno-deficiency; TTL, time to leukemia.

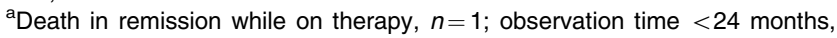
$n=1$.

peripheral blood, bone marrow and spleen by flowcytometry demonstrating advanced human ALL in the mice.

The time between the transplantation of primary patient cells and manifestation of overt ALL was estimated for each leukemia transplanted (TTL). In this cohort of 23 leukemia

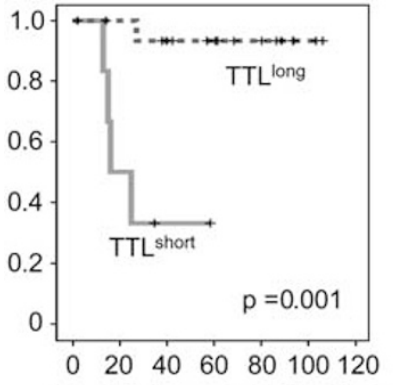

relapse free survival [months]

Figure 1 Rapid NOD/SCID/huALL engraftment is associated with inferior patient outcome. Superior relapse-free survival of patients whose ALL cells engraft in NOD/SCID recipients with a TTL ${ }^{\text {long }}$ phenotype compared with TTL $L^{\text {short }}$ patients. Kaplan-Meier analysis, $P=$ significance by log-rank test

samples, 7 led to ALL manifestation in the recipient animals after a short period of time of $<10$ weeks (TTL ${ }^{\text {short}}$ ) and 16 showed prolonged NOD/SCID engraftment ( $T T^{\text {long}}{ }^{\text {) }}$. Patients whose leukemia samples exhibited a $\mathrm{TTL}^{\text {short }}$ phenotype showed inferior survival in contrast to patients with late leukemia onset in NOD/SCID mice (Figure 1; TTL ${ }^{\text {short }}$ $n=7$, mean relapse-free patient survival: 30.9 months; $\mathrm{TTL}^{\text {long }} n=16$, mean relapse-free patient survival: 100.8 months; log-rank $P=0.001$ ) also in this cohort of patients, in line with our earlier findings. ${ }^{5}$

Prolonged NOD/SCID engraftment is associated with high death-associated protein kinase1 (DAPK1) expression. Previously, we analyzed global gene expression profiles of both engraftment phenotypes and identified pathways pointing to dysregulated apoptosis signaling in TTL ${ }^{\text {short} / e a r l y-r e l a p s e ~ l e u k e m i a . ~ T w o ~ c a n d i d a t e ~ g e n e s ~ c o d-~}$ ing for molecules involved in cell death signaling were identified in our previous study to be significantly differentially regulated: DAPK1 and phosphodiesterase $4 \mathrm{~A}(P D E 4 A)$, and were now investigated further in this study. Transcript levels of both genes were analyzed by quantitative polymerase chain reaction (PCR) and compared in TTL-subgroups. DAPK1, which codes for the pro-apoptotic death-associated kinase $1^{9}$ was found to be downregulated in $\mathrm{TTL}^{\text {short }}$ compared with

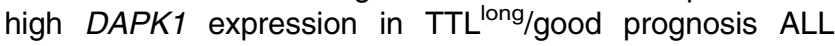
$(N=23$, $T$-test, $P=0.005$; Figure 2a) and increasing $D A P K 1$ transcript levels are significantly associated with prolonged TTL (Spearman correlation, $N=23, r_{\mathrm{s}}=.648, P=0.001$ ). Moreover, we also analyzed DAPK1 protein expression. Western blot analysis showed expression of DAPK1 protein in $T_{T L}$ long but not $T L^{\text {short }}$ leukemia samples (Figure $2 b$ ), resulting in significant higher protein expression levels of this pro-apoptotic molecule in $\mathrm{TTL}^{\text {long/favorable outcome } \mathrm{ALL}}$ $(N=23, T$-test, $P=0.005$; Figure $2 \mathrm{c})$. Alike transcript levels, increasing DAPK1 protein expression is significantly correlated with prolonged TTL (Spearman correlation, $N=23$, $\left.r_{\mathrm{s}}=.427, P=0.04\right)$. These results confirm our previous gene expression array data reflecting DAPK1 upregulation in $\mathrm{TTL}^{\text {long }}$ samples on protein level.

$P D E 4 A$, coding for a cyclic adenosine monophosphate phosphodiesterase mediating reduced apoptosis sensitivity ${ }^{10}$ exhibited elevated transcript levels in $T_{T L}^{\text {short }}$ leukemias. 

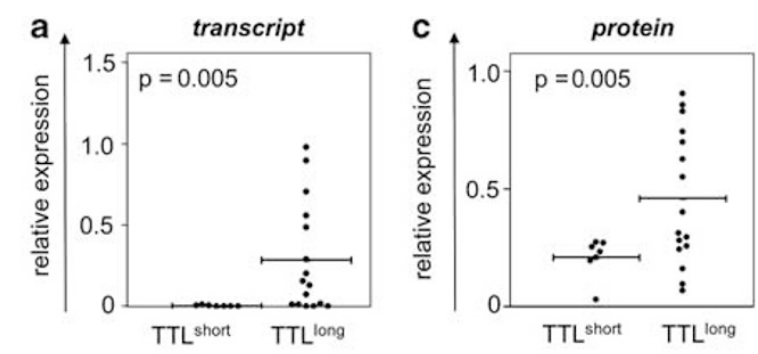

b

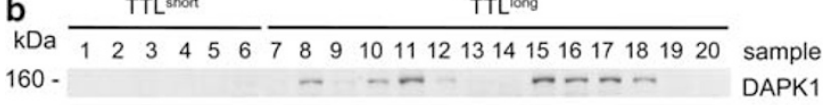

41- - - - -

$$
\begin{aligned}
& \text { TTL short } \frac{T T L^{\text {long }}}{\mathrm{kDa}}-\frac{\mathrm{T}}{2122} \text { sample } \\
& 160-\quad \text { DAPK1 } \\
& 41-\longrightarrow \text { ACTIN }
\end{aligned}
$$

Figure 2 The TTL long phenotype is associated with high DAPK1 expression. (a) Distinct DAPK1 transcript expression in ALL xenografts $(N=23)$ with a TTL ${ }^{\text {shor }}$ $(n=7)$ compared with a TTL long $(n=16)$ phenotype; bars represent mean gene expression, $T$-test, $P=$ significance. (b) DAPK1 western blot analysis of $N=23$ ALL xenografts showing the presence of DAPK1 protein in TTL long but not TTL short samples. (c) Higher DAPK1 protein expression (relative to beta-Actin) in TTL ${ }^{\text {long }}$ ALL; bars represent mean protein expression, $T$-test, $P=$ significance

However, the difference was not significant in this study cohort including leukemia samples carrying ETV6/RUNX1 (ETS (E-twenty six) variant 6/runt-related transcription factor 1 ), $\mathrm{BCR} / \mathrm{ABL}$ (breakpoint cluster region/abelson oncogene) gene fusions or MLL-(mixed lineage leukemia)-rearrangements, which were not present in our previous analysis. Nevertheless, if ALL samples with these gene fusions were not included into the analysis, $P D E 4 A$ was significantly upregulated in $\mathrm{TTL}^{\text {short }}$ leukemia ( $T$-test, $N=16$, $\mathrm{TTL}^{\text {short }} n=6$, $\left.\mathrm{TTL}^{\text {long }} n=10, P=0.024\right)$.

Enhanced apoptosis signaling in $\mathrm{TTL}^{\text {long }}$ leukemia. Mitochondrial release of apoptogenic molecules, particularly cytochrome c (cc) and the subsequent formation of the apoptosome complex which in turn leads to caspase activation and ultimately apoptosis execution is a key event in downstream apoptosis signaling. To address the efficacy of the apoptosis machinery, we determined the functional ability of leukemia cells to undergo apoptosis. Two key apoptogenic events were analyzed in xenograft leukemia samples: mitochondrial release of $\mathrm{cc}$ and activation of the downstream caspase-3 (ac). Leukemia cells were isolated from leukemia-bearing recipients and further investigated before $(0 \mathrm{~h})$ and after $(16 \mathrm{~h})$ ex vivo culture for the induction of spontaneous apoptosis. Leukemia cells were stained with antibodies specifically binding to cc and the active caspase-3 fragment and analyzed by flowcytometry. Cell death was evaluated according to forward/side scatter criteria. Cytochrome $c$ release and the consecutive apoptosome formation induce activation of effector caspases such as caspase-3 (Figure $3 a$ ), resulting in cells staining low for $\mathrm{cc}$ and positive for active caspase-3 after culture (Figure 3bi). In contrast, impaired cc release and lack of caspase- 3 activation imply disturbed apoptosomal function and defect apoptosis signaling (Figure 3bii).

Overall cd, release of cc and caspase-3 activation was assessed for all xenograft ALL samples and compared in both TTL-subgroups. All apoptosis parameters were found to be higher in TTL ${ }^{\text {long }}$ leukemia with statistical significant higher

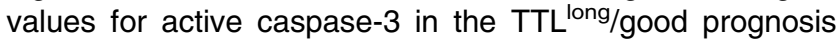
subgroup (Table 2). Most interestingly, if directly correlated to TTL, we found that increased cd and ac are significantly associated with longer TTL (Figure 3ci and ii). Thus, high activity of executor caspases is characteristic for TTL ${ }^{\text {long/ }}$ favorable prognosis ALL.

Moreover, in addition to functional apoptosis signaling, we analyzed whether molecules negatively regulating apoptosome formation (Bcl-2 and $\mathrm{Mcl}-1$ ) or function (XIAP and Livin) would be downregulated in $\mathrm{TTL}^{\text {long }}$ leukemia characterized by activated apoptosis signaling. However, gene expression of $B c l-2, M c l-1, X I A P$ and Livin was not significantly lower in $\mathrm{TTL}^{\text {long }}$ compared with $\mathrm{TTL}^{\text {short }}$ samples. In line with the transcript data, western blot analysis did not show significant differences in Bcl-2, Mcl-1, XIAP and Livin protein expression with respect to TTL-subgroups (Figure 4).

Proficient apoptosome function is associated with prolonged engraftment and favorable outcome. Concomitant cc release and caspase-3 activation corresponds to apoptosome formation and function and implies intact apoptosis signaling. The functional integrity of this apoptosis checkpoint is reflected by a significant correlation of $\mathrm{cc}$ and $\mathrm{ac}^{11,12}$ and was exclusively detected in xenograft leukemia derived from patients with good response to treatment, stratification into non-high-risk groups, and from non-relapse patients. Most importantly, this significant association was also observed in leukemia samples showing a long time to leukemia (TTL ${ }^{\text {long }}$ ) engraftment phenotype (Table 3$)$. Conversely, this correlation was completely absent in xenografts derived from patients who did not achieve early remission, were stratified into high-risk groups, and who encountered relapse. In contrast to $\mathrm{TTL}^{\text {long }}$ xenografts showing concomitant cc release and caspase-3 activation, these two apoptogenic events were not associated in xenograft ALL with a TTL ${ }^{\text {short }}$ phenotype.

In addition, the same significant correlation of released cc and active capase- 3 was observed in xenograft ALL samples showing high transcript and protein expression levels of proapoptotic DAPK1 and deficient apoptosis signaling was associated with low DAPK1 expression (Table 3).

Taken together, this indicates that deficient apoptosome formation and function in B-cell precursor (BCP)-ALL cells is associated with downregulation of the positive apoptosis regulator DAPK1, rapid NOD/SCID/huALL engraftment, and poor patient prognosis and outcome.

Proficient apoptosis signaling in ALL xenografts predicts patient outcome. Intact or defective apoptosis signaling is characterized by synchronous or dysregulated cc release and caspase-3 activation. For an individual leukemia sample, the effectivity of apoptosis signaling can be assessed by the 


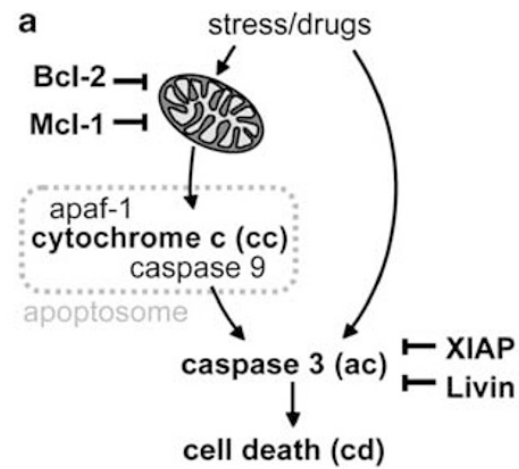

b i

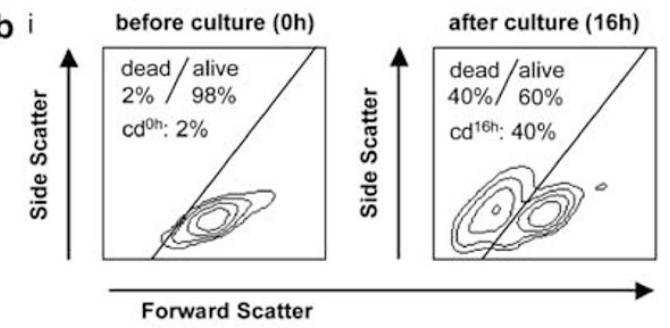

ii before culture $(0 \mathrm{~h})$

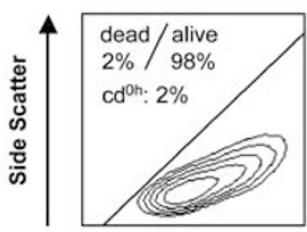

Forward Scatter

C

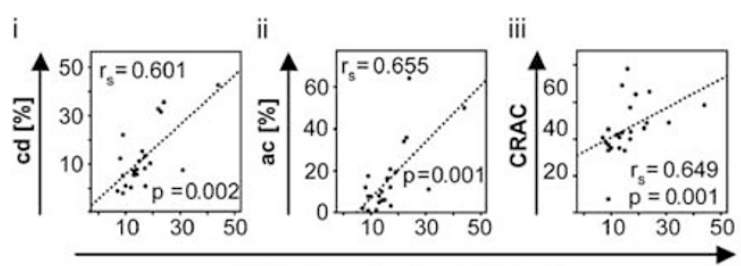

TTL (time to leukemia) [weeks]
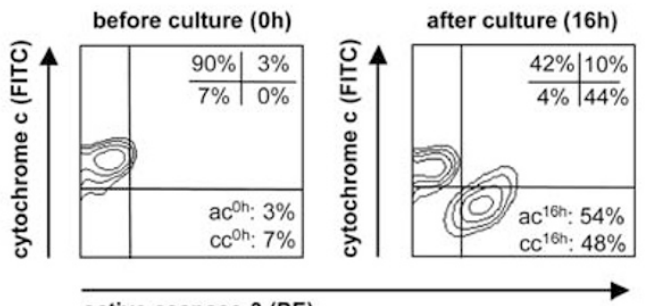

active caspase-3 (PE)
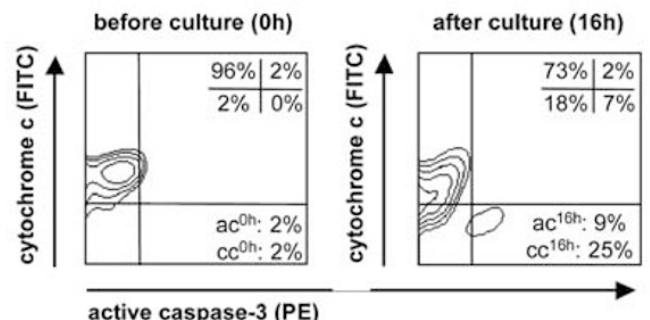

d

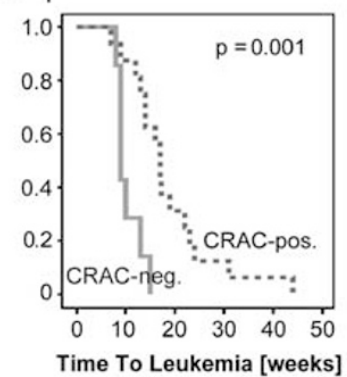

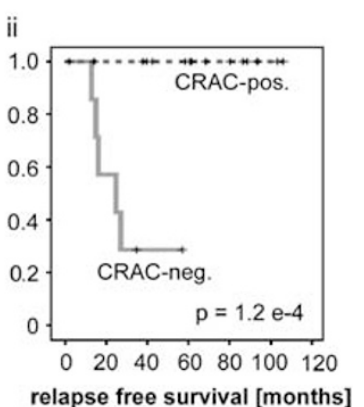

Figure 3 Analysis of apoptosis signaling in xenograft ALL and association with patient outcome. (a) Analysis of cd parameters and anti-apoptotic molecules acting on mitochondria or downstream effector caspases. (b) Flowcytometric analysis of cd according to forward/side scatter properties, and of ac and cc release (cc) by simultaneous intracellular staining. (i) proficient signaling as indicated by a positive CRAC value $\left(a c=a c^{16 \mathrm{~h}}-\mathrm{ac}^{0 \mathrm{~h}}=54 \%-3 \%=51 \%\right.$; $c \mathrm{c}=\mathrm{cc} \mathrm{c}^{16 \mathrm{~h}}-\mathrm{cc} \mathrm{c}^{0 \mathrm{~h}}=$ $48 \%-7 \%=41 \%$; CRAC $=a c-c c=51 \%-41 \%=+10$. (ii) deficient signaling as indicated by a negative CRAC value (ac $=9 \%-2 \%=7 \% ; c c=25 \%-2 \%=23 \%$; $\mathrm{CRAC}=\mathrm{ac}-\mathrm{cc}=7 \%-23 \%=-16$. (c) TTL significantly correlates to (i) cd; (ii) ac, and (iii) cc-related activation of caspase-3 (CRAC). Spearman correlation, $r_{\mathrm{s}}=$ correlation coefficient, $P=$ significance. (d) Prolonged NOD/SCID/huALL engraftment of patient leukemia cells (i) and superior relapse-free survival of patients with proficient apoptosis signaling (CRAC-positive) in the corresponding xenograft samples (ii). $N=23$, Kaplan-Meier analyses, $P=$ significance by log-rank test

parameter 'cc-related activation of caspase-3 (CRAC)', which is calculated as the difference of ac and cc release. ${ }^{11}$ A positive CRAC value indicates proficient signaling, whereas a negative value results in deficient apoptosis signaling (Figure 3bi and ii). We calculated the values for ccrelated activation of caspase-3 (CRAC) for each of the 23 xenograft ALL samples and found that TTL long leukemia is characterized by positive CRAC values, whereas TTL ${ }^{\text {short }}$ showed significantly lower, negative CRAC values (Table 2).
Interestingly, an incremental propensity of the leukemia cells to undergo apoptosis indicated by increasing CRAC values was significantly associated with prolonged TTL (Figure 3ciii).

We then compared CRAC-negative $(n=7)$ and CRACpositive $(n=16)$ leukemia samples with respect to their TTL after xenotransplantation. Most interestingly, CRAC-negative leukemias (deficient apoptosis signaling) showed a significantly shorter TTL in contrast to CRAC-positive samples 
Table 2 Apoptosis signaling parameters in $T T L^{\text {long }}$ and $T T L^{\text {short }}$ leukemia subgroups

\begin{tabular}{|c|c|c|c|c|c|c|c|c|c|c|c|c|}
\hline & \multicolumn{3}{|c|}{ cd } & \multicolumn{3}{|c|}{ ac } & \multicolumn{3}{|c|}{ cc } & \multicolumn{3}{|c|}{ CRAC } \\
\hline & Mean & S.E.M. & $\boldsymbol{P}$ & Mean & S.E.M. & $\boldsymbol{P}$ & Mean & S.E.M. & $\boldsymbol{P}$ & Mean & S.E.M. & $\boldsymbol{P}$ \\
\hline TTL & & & 0.078 & & & $0.029^{a}$ & & & 0.394 & & & $0.011^{a}$ \\
\hline long & 14.7 & 3.3 & & 19.1 & 4.5 & & 13.6 & 4.1 & & 5.5 & 1.6 & \\
\hline short & 6.1 & 3.2 & & 7.1 & 2.4 & & 8.7 & 3.8 & & -3.0 & 2.3 & \\
\hline
\end{tabular}

Abbreviations: ac, activated caspase-3; cc, cytochrome c release; cd, cell death; CRAC, cytochrome c-related activation of caspase-3; S.E.M., standard error of the mean; $P$, significance.

${ }^{\mathrm{a}}$ Difference is significant.

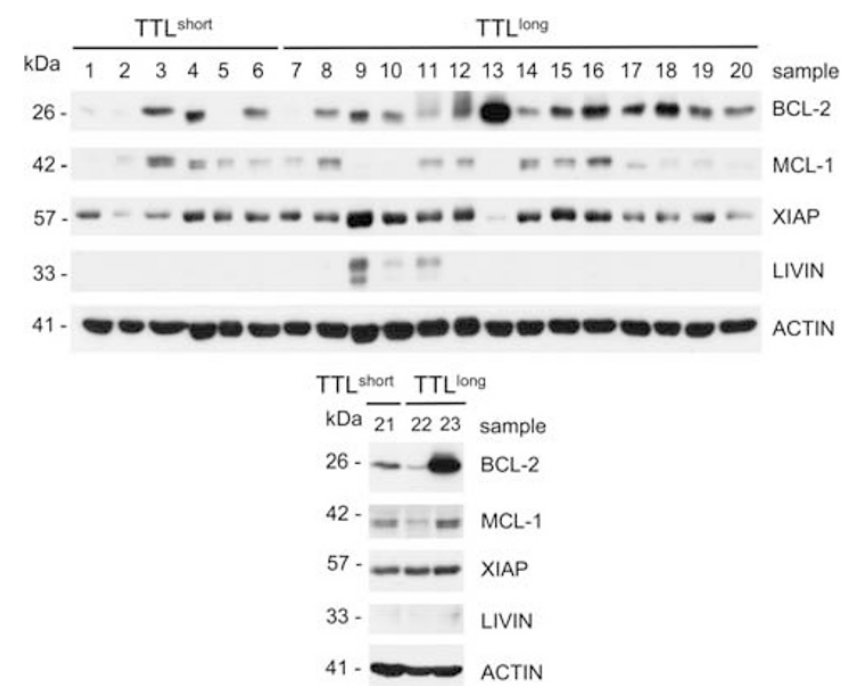

Figure 4 Similar Bcl-2, Mcl-1, XIAP and Livin protein levels in TTL long and $T T L^{\text {short }}$ xenograft ALL. Western blot analysis of ALL xenografts $(N=23)$ showing no distinct protein expression in TTL ${ }^{\text {long }}$ versus $T T L^{\text {short }} \mathrm{ALL}$ for $\mathrm{Bcl}-2(P=0.053)$, Mcl-1 $(P=0.772), \operatorname{XIAP}(P=0.093)$ and Livin $(P=0.155 ; T$-test, $P=$ significance; densitometric quantification of protein expression relative to beta-Actin)

(mean TTL, CRAC-negative: 10.4 weeks; CRAC-positive: 18.7 weeks; log-rank $P=0.001$; Figure 3di), indicating that disturbed apoptosis signaling of the leukemia cells has an impact on NOD/SCID engraftment.

Furthermore, we analyzed relapse-free patient survival according to positive or negative cc-related activation of caspase-3 (CRAC) as measured in the corresponding xenograft ALL samples. Most importantly, patients with negative CRAC signifying deficient apoptosomal function showed an inferior outcome in contrast to $100 \%$ survival of CRAC-positive patients featuring intact signaling in xenograft leukemia cells (log-rank $P=1.2 \mathrm{e}-4$; Figure 3dii).

Taken together, leukemia apoptosis sensitivity contributes to both, the TTL phenotype in NOD/SCID/huALL, a feature associated with early relapse, and patient survival.

\section{Discussion}

In this study, we analyzed the function of apoptosis signaling in xenograft ALL samples engrafted in our model of NOD/ SCID/huALL. Intact apoptosome formation and function reflecting an efficient distal signaling machinery implies
Table 3 Intact cytochrome c-related caspase activation in favorable ALL

\begin{tabular}{|c|c|c|c|}
\hline & $n$ & $r_{\mathrm{s}}$ & $\boldsymbol{P}$ \\
\hline \multicolumn{4}{|c|}{ Remission on day 15} \\
\hline Yes & 7 & $0.893^{\mathrm{a}}$ & 0.007 \\
\hline No & 7 & 0.643 & 0.119 \\
\hline \multicolumn{4}{|c|}{$A L L-B F M$ risk group } \\
\hline Non-HR & 19 & $0.793^{\mathrm{a}}$ & $<0.001$ \\
\hline $\mathrm{HR}$ & 4 & -0.400 & 0.600 \\
\hline \multicolumn{4}{|l|}{ Relapse } \\
\hline No & 16 & $0.759^{\mathrm{a}}$ & 0.001 \\
\hline Yes & 5 & 0.600 & 0.285 \\
\hline \multicolumn{4}{|c|}{ Time to leukemia } \\
\hline TTL long & 16 & $0.750^{\mathrm{a}}$ & 0.001 \\
\hline TTL ${ }^{\text {short }}$ & 7 & 0.750 & 0.052 \\
\hline \multicolumn{4}{|c|}{ DAPK1, transcript expression } \\
\hline High & 11 & $0.791^{\mathrm{a}}$ & 0.004 \\
\hline Low & 12 & 0.517 & 0.085 \\
\hline \multicolumn{4}{|c|}{ DAPK 1 , protein expression } \\
\hline High & 11 & $0.791^{\mathrm{a}}$ & 0.004 \\
\hline Low & 12 & 0.517 & 0.085 \\
\hline
\end{tabular}

Abbreviations: ALL, acute lymphoblastic leukemia; BFM, Berlin Frankfurt Münster study group; DAPK1, death-associated protein kinase1; HR, high risk; TTL, time to leukemia.

Spearman correlation, $r_{\mathrm{s}}$ indicates correlation coefficient; $P$, significance.

${ }^{a}$ Correlation is significant.

prolonged NOD/SCID engraftment, good response to treatment, favorable prognosis and relapse-free patient survival.

Consistent with earlier reports, the established BCP-ALL xenografts resembled the human disease with infiltration of leukemia cells in peripheral blood, bone marrow and spleen of the recipient animals. ${ }^{13,14}$ Individual patient ALL samples led to manifestation of overt leukemia after different periods of time. A short time from transplantation until onset of xenograft leukemia (TTL) is associated with inferior relapse-free patient survival as described previously. ${ }^{5}$ In line, distinct engraftment phenotypes of individual patient ALL samples transplanted into xenograft mouse models similar to the NOD/SCID/huALL model used in our study were observed ${ }^{15}$ and associated with poor initial patient response. ${ }^{16}$

Characterization of the distinct engraftment phenotypes by genome wide expression analysis revealed genes coding for molecules involved in apoptosis regulation pointing to ineffective apoptosis signaling in TTL ${ }^{\text {short}} /$ poor outcome ALL. ${ }^{5}$ We quantified the expression of two previously identified candidate genes in this study cohort. High transcript and 
protein expression of the positive apoptosis regulator DAPK1 is characteristic for TTL long leukemia and was associated with prolonged TTL confirming our earlier array data. Antiapoptotic PDE4A showed upregulated transcripts in $\mathrm{TTL}^{\text {short }}$ $A L L$, although not reaching statistical significance in the whole-study cohort. However, consistent to our previous data obtained from leukemia samples without gene fusions, a significantly higher PDE4A expression was observed in the subset of samples negative for known gene fusions indicating that expression of PDE4A is heterogeneously regulated and might be influenced by specific gene expression profiles characteristic for ALL subgroups with gene fusions. ${ }^{17,18}$

To evaluate the functional relevance of efficient or altered apoptosis signaling on NOD/SCID/huALL engraftment, we investigated apoptosome formation and function in xenograft samples. Furthermore, gene expression and protein levels of negative regulators acting on this distal part of the signaling pathway were analyzed. However, we found no downregulated expression of the anti-apoptotic molecules Bcl-2, Mcl-1, XIAP

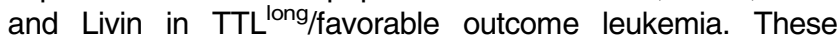
findings are in line with earlier reports on a lack of correlation between $\mathrm{Bcl}-2$ or $\mathrm{Mcl}-1$ gene expression in leukemia cells and patient outcome. ${ }^{19-23}$ Counterintuitively to its anti-apoptotic function, high Livin expression was related to relapse-free survival in pediatric ALL patients. ${ }^{24}$ However, we did not observe an association of Livin transcript levels and NOD/SCID engraftment. Alike our results in ALL xenografts, XIAP expression was shown to have no impact on outcome in acute myeloid leukemia (AML) patients. ${ }^{25}$ On the contrary, high XIAP transcript levels were reported to be associated with poor prognosis in pediatric and adult AML patients. ${ }^{22,26,27}$ Expression of apoptosis-regulating molecules has been addressed in several studies on acute leukemia, not demonstrating clear impact with respect to prognosis and outcome even showing contradictory results. For instance, expression of the proapoptotic molecule $\mathrm{Bax}$ (Bcl-2-associated $\mathrm{X}$ protein) was reported to be associated with good, ${ }^{21}$ poor $^{28}$ or not to be associated $^{23,27}$ with prognosis in acute leukemia. Consequently, expression of single molecules will not reflect the efficacy of the apoptosis machinery and determine the functional ability of a leukemia cell to undergo apoptosis.

To address this issue, we investigated apoptosis signaling on a functional level and analyzed overall cd and two key apoptogenic events of the distal apoptosis pathway, mitochondrial cc release and caspase- 3 activation. Interestingly, increased cd and ac were significantly associated with prolonged TTL. Moreover, concomitant cc release and caspase activation implying effective apoptosome formation and function is a feature of xenograft ALL derived from patients with good response to treatment and who do not encounter relapse. Importantly, favorable ALL with a TTL long phenotype is characterized by effective apoptosis signaling in contrast to deficient signaling in TTL short/poor prognosis ALL. Likewise, high caspase-3 activity and proficient distal apoptosis signaling was described in primary leukemia cells of patients with good prognosis and superior outcome in ALL and AML. ${ }^{11,12,29}$ Furthermore, leukemias with high gene and protein expression of DAPK1, a pro-apoptotic molecule that suppresses in vivo tumor growth ${ }^{9,30,31}$ and was previously identified to be upregulated in $\mathrm{TTL}^{\text {long }}$ phenotype leukemias, showed synchronous cc release and caspase activation indicating apoptosomal function in contrast to low DAPK1 associated with deficient signaling. High transcript expression of $\mathrm{Bcl}-2, \mathrm{Mcl}-1, \mathrm{XIAP}$ and Livin was found in ALL cells with defective downstream apoptosis signaling but no association was observed with respect to the protein levels of the molecules (data not shown). Along this line, downregulated $\mathrm{Bcl}-2, \mathrm{Mcl}-1$ and Livin transcripts were described in ALL cells showing in vitro drug sensitivity ${ }^{32}$ in contrast to other reports on no association of $\mathrm{Bcl}-2$ and $\mathrm{Mcl}-1$ protein expression with in vitro drug sensitivity and patient outcome in pediatric $\mathrm{ALL}^{19,20}$ and absent impact of XIAP protein levels on AML patient survival. ${ }^{25}$ This emphasizes again the importance to functionally investigate key signaling checkpoints on which different signaling pathways such as cc, caspase-3 and apoptosome formation converge.

Evaluation of the prognostic impact of cc-related caspase activation requires analysis of apoptosis signaling in the individual leukemia sample. Proficient or deficient function of this downstream apoptosis signaling checkpoint is subsumed by the parameter 'cc-related activation of caspase-3 (CRAC)'. CRAC-positive leukemias show a significantly longer time from transplantation to ALL manifestation in the recipient mice compared with CRAC-negative cells. The engraftment efficacy of xenotransplanted human leukemia cells is determined by several factors including the ability of the cells to survive in the inhospitable environment of the recipient. ${ }^{33}$ This suggests that apoptosis deficiency confers prolonged leukemia cell survival and thereby an advantage for xenotransplanted CRAC-negative leukemia cells to overcome the recipients hostility leading to rapid ALL manifestation ( $T T L^{\text {short }}$ ), in contrast to inferior engraftment of CRAC-positive leukemia cells showing intact apoptosis signaling and ultimately a long TTL. Most importantly, patients with CRAC-positivity as assessed in the corresponding xenograft leukemia samples showed $100 \%$ survival without relapse in contrast to patients whose xenograft counterparts exhibit CRAC-negativity. This is in line with our earlier findings on apoptosis deficiency (negative CRAC) identified in patient leukemia cells obtained at diagnosis and inferior patient survival in ALL and AML. ${ }^{11,12}$ Moreover, this indicates that in addition to mimicking disease distribution and leukemia characteristics, ${ }^{5,34-36}$ ALL xenografts also recapitulate features of leukemia biology such as proficient or deficient apoptosis signaling.

Taken together, leukemia apoptosis sensitivity contributes to both, the TTL phenotype in NOD/SCID/huALL, a feature associated with early relapse, and overall survival highlighting cd and survival pathways as important targets for therapeutic intervention strategies.

\footnotetext{
Materials and Methods

Xenograft ALL. Twenty-three patient derived xenograft ALL samples established in our NOD/SCID/huALL xenotransplant mouse model were investigated in this study. Xenotransplant experiments were approved by the appropriate authority and performed as described earlier. ${ }^{5}$ In brief, leukemia cells $\left(1 \times 10^{7}\right.$ per transplant) obtained from the patient at diagnosis or cryopreserved ALL leukemia cells of previously established xenograft leukemias were intravenously injected into

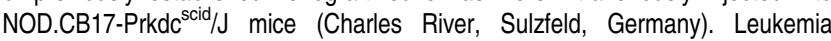
samples of pediatric patients diagnosed with de novo BCP-ALL were obtained after informed consent in accordance with the institution's ethical review board. Patients were treated within the ALL-Berlin Frankfurt Münster study group 2000
} 
protocol ${ }^{4}$ in conformance to the Helsinki Declaration (http://clinicaltrials.gov: NCT00430118). Twelve of the leukemia samples have been investigated within previous studies on NOD/SCID engraftment. After transplantation, recipient animals were regularly examined and killed at manifestation of leukemia-related morbidity. TTL was estimated for each patient sample transplanted as weeks from transplantation to manifestation of overt leukemia. Autopsy was performed and the presence of high percentage human ALL infiltration was confirmed by flowcytometry staining for huCD19 or huCD45 in peripheral blood and cell suspensions prepared from spleen and bone marrow. Patient and xenograft characteristics are summarized in Table 1.

Analysis of apoptosis signaling. Xenograft leukemia cells were isolated from ALL-bearing mice and analyzed for apoptosis signaling. Cell suspensions prepared from spleens of leukemia-bearing recipients contained at least $80 \%$ ALL cells as estimated by flowcytometry staining for human CD45 or CD19. Spontaneous apoptosis was induced in xenograft leukemia cells by factor deprivation in culture, assessed by flowcytometry and evaluated in the cc versus caspase-3 plot quantifying the parameters $\mathrm{cd}$, cc release and ac as described in detail before. ${ }^{11,12,37}$ In brief, spontaneous apoptosis was induced in xenograft ALL cells directly after isolation from leukemia-bearing recipients by culture for 16 h (RPMI 1640 medium, supplemented with 10\% fetal calf serum, penicillin, streptomycin and glutamine at $37^{\circ} \mathrm{C}$ in humidified air with $5 \%$ carbon dioxide). Primary BCP-ALL cells depend on cell-cell interaction and cannot be kept viable in vitro by growth factors. ${ }^{38,39}$ Exposure of primary xenograft BCP-ALL cells to culture conditions lacking cellular survival signals provides a stimulus inducing spontaneous apoptosis by a 'death by default' mechanism.

Cells were stained intracellularly with anti-cytochrome c (primary, clone 7H8.2C12, BD Bioscience, Heidelberg, Germany, and secondary goat anti-mouse IgG2b fluorescein isothiocyanate conjugated, Southern Biotech, Birmingham, AL, USA) and anti-active caspase-3 (phycoerythrine conjugated, BD Bioscience) antibodies and analyzed on a FACSCalibur flowcytometer using Cell Quest software (both from BD Bioscience). Experiments were performed in triplicate and mean values were used for subsequent analysis. cd was measured according to forward and side scatter criteria. cc release and caspase-3 activation were evaluated in the cc versus caspase-3 plot (Figure $3 b$ ): ac was quantified as percentage of events in the upper and lower right quadrant, and cc release was detected by reduction of the cc signal and calculated as percentage of events in the lower left and right quadrants. Absolute values were estimated as the difference of single values obtained at $16 \mathrm{~h}$ and $0 \mathrm{~h}$. cc-related activation of caspase-3 (CRAC) was calculated as the difference of $\mathrm{ac}$ and $\mathrm{cc}(\mathrm{ac}-\mathrm{cc})$. Equal or higher values of caspase-3 activation compared with cc release result in positive CRAC values and indicate proficient signaling (Figure 3bi). Deficient caspase activation despite released $\mathrm{CC}$ results in negative CRAC values indicating defective apoptosis signaling (Figure 3bii).

Analysis of gene expression. Transcripts of DAPK1, PDE4A, Mcl-1, $B C l-2, X I A P$ and Livin were analyzed by quantitative real-time PCR (LightCycler, Fast Start DNA Master SYBR Green I kit, Roche, Grenzach-Wyhlen, Germany) and normalized to succinate dehydrogenase A (SDHA), TATA box-binding protein (TBP) and beta-2-microglobulin (B2M). High/low transcript levels were determined according to the median expression. The following primers were used:

DAPK1, sense: 26s: 5'-CGAGGTGATGGTGTATGGTG-3';

DAPK1, antisense: 26a: 5'-CTGTGCTTTGCTGGTGGA-3';

PDE4A sense: 14s: 5'-GCAGTGTTCACGGACCTGGAGATTCTCGC-3';

PDE4A antisense: 16a: $5^{\prime}$-GCGGTCGGAGTAGTTATCTAGCAGGAGGACCCC-3';

Bcl-2 sense: 1s: $5^{\prime}$-CCTTCACCGCGCGGGGACGCTTTG-3';

Bcl-2 antisense: 2a: 5'-GATAGGCACCCAGGGTGATGCAAGC-3';

Mcl-1 sense: 2s: 5'-CGAAGACGATGTGAAATCGTTGTCTCGAGTG-3';

Mcl-1 antisense: 3a: 5'-GATATGCCAAACCAGCTCCTACTCCAGCAACA-3';

XIAP (BIRC4) sense: 3s: 5'-GATTGGAAGCCCAGTGAAGACCCTTGGG-3';

XIAP (BIRC4) antisense: 6a: 5'-CTTAATGTCCTTGAAACTGAACCCCATTCG-3';

Livin (BIRC7) sense: 1s: 5'-GCATGGGCTCTGAGGAGTTGCGTCTG-3';

Livin (BIRC7) antisense: 3a: 5'-GCAGCTGGGAGTGAGTCTCCTGCACACTG-3';

SDHA sense: 12s: $5^{\prime}$-CATGCTGCCGTGTTCCGTGTGGG-3';

SDHA antisense: 14a: 5'-GGACAGGGTGTGCTTCCTCCAGTGCTCC-3';

TBP sense: 2s: $5^{\prime}$-GAGGAAGTTGCTGAGAAGAGTGTGCTGGAG-3';

TBP antisense: 3a: 5'-GTCAGTCCAGTGCCATAAGGCATCATTGG-3';

B2M sense: 2s: 5'-GTGGAGCATTCAGACTTGTCTTTCAGCAAGGAC-3';

B2M antisense: 2a: 5'-CACTTACTATCTTGGGCTGTGACAAAGTCACATGG-3'.
Analysis of protein expression. Protein expression of DAPK1, Bcl-2, $\mathrm{Mcl}-1, \mathrm{XIAP}$ and Livin was analyzed by western blot analysis. Cells were lysed for $15 \mathrm{~min}$ at $4{ }^{\circ} \mathrm{C}$ in lysis buffer (tris-HCL $30 \mathrm{mM}, \mathrm{NaCl} 150 \mathrm{mM}$, tritonX $1 \%$ and glycerol $10 \%)$ and then underwent high-speed centrifugation. Protein concentration was assessed using bicinchoninic acid (Thermo Scientific, Waltham, MA, USA); $40 \mu \mathrm{g}$ protein per lane was separated by $12 \%$ sodium dodecyl sulfate-polyacrylamide gel electrophoresis and electroblotted onto nitrocellulose (Amersham GE Healthcare, Piscataway, NJ, USA). After blocking for $1 \mathrm{~h}$ in phosphate-buffered saline supplemented with $5 \%$ milk and $0.1 \%$ Tween 20 (Sigma-Aldrich, St. Louis, MO, USA), immunodetection was performed using the following antibodies: mouse antiDAPK1 monoclonal antibody (Sigma-Aldrich), mouse anti-Bcl-2 monoclonal antibody (BD Bioscience), rabbit anti-Mcl-1 polyclonal antibody (Stressgen, Victoria, BC, Canada), mouse anti-XIAP monoclonal antibody (BD Bioscience), mouse anti-Livin monoclonal antibody (Imgenex, St. Diego, CA, USA) and mouse anti-beta-Actin monoclonal antibody (Sigma-Aldrich), followed by goat anti-mouse lgG or goat antirabbit IgG conjugated to horseradish peroxidase (Santa Cruz Biotechnology, Santa Cruz, CA, USA). Enhanced chemiluminescence was used for detection (Thermo Scientific). Specificity of bands on western blots was identified by molecular weight markers. Densitometric quantification was performed using ImageJ $1.45 \mathrm{~s}$ software. ${ }^{40}$

Statistical analysis. Analyses were carried out using SPSS 19.0 software (IBM, Munich, Germany) using the respective tests indicated, $P$ values of $<0.05$ were considered significant in all tests performed in this study.

\section{Conflict of Interest}

The authors declare no conflict of interest.

Acknowledgements. This work was supported by the program 'Experimental Medicine' of the International Graduate School in Molecular Medicine, University of Ulm (FS); Kind-Philipp-Foundation for Leukemia Research (SU), Else Kröner-Fresenius-Foundation (MS); German Research Foundation (Deutsche Forschungsgemeinschaft) (K-MD; STA555-3); Wilhelm Sander Foundation (K-MD; 2005.075.2); and a research fellowship of the European Hematology Association (LHM). We thank Manuel Hermann, Simone Miller and Susann Baumgart for their excellent technical assistance.

1. Pui CH, Evans WE. Treatment of acute lymphoblastic leukemia. N Engl J Med 2006; 354: 166-178

2. Schrappe M, Reiter A, Ludwig WD, Harbott J, Zimmermann M, Hiddemann W et al Improved outcome in childhood acute lymphoblastic leukemia despite reduced use of anthracyclines and cranial radiotherapy: results of trial ALL-BFM 90. German-AustrianSwiss ALL-BFM Study Group. Blood 2000; 95: 3310-3322.

3. Henze G, Fengler R, Hartmann R, Kornhuber B, Janka-Schaub G, Niethammer D et al. Six-year experience with a comprehensive approach to the treatment of recurrent childhood acute lymphoblastic leukemia. Blood 1991; 78: 1166-1172.

4. Conter V, Bartram CR, Valsecchi MG, Schrauder A, Panzer-Grumayer R, Moricke A et al. Molecular response to treatment redefines all prognostic factors in children and adolescents with B-cell precursor acute lymphoblastic leukemia: results in 3184 patients of the AIEOP-BFM ALL 2000 study. Blood 2010; 115: 3206-3214.

5. Meyer LH, Eckhoff SM, Queudeville M, Kraus JM, Giordan M, Stursberg J et al. Early relapse in all is identified by time to leukemia in nod/scid mice and is characterized by a gene signature involving survival pathways. Cancer Cell 2011; 19: 206-217.

6. Hanahan D, Weinberg RA. Hallmarks of cancer: the next generation. Cell 2011; 144: 646-674.

7. Suminoe A, Matsuzaki A, Hattori $\mathrm{H}$, Koga $\mathrm{Y}$, Kinukawa $\mathrm{N}$, Ishii $\mathrm{E}$ et al. mRNA expression of apoptosis-associated genes in infant acute lymphoblastic leukemia: low Fas expression is an independent predictor for poor prognosis. Leukemia 2004; 18: 365-368.

8. Del Poeta G, Venditti A, Del Principe MI, Maurillo L, Buccisano F, Tamburini A et al. Amount of spontaneous apoptosis detected by Bax/Bcl-2 ratio predicts outcome in acute myeloid leukemia (AML). Blood 2003; 101: 2125-2131.

9. Deiss LP, Feinstein E, Berissi H, Cohen O, Kimchi A. Identification of a novel serine/ threonine kinase and a novel $15-\mathrm{kD}$ protein as potential mediators of the gamma interferoninduced cell death. Genes Dev 1995; 9: 15-30.

10. Jiang X, Li J, Paskind M, Epstein PM. Inhibition of calmodulin-dependent phosphodiesterase induces apoptosis in human leukemic cells. Proc Natl Acad Sci USA 1996; 93: 11236-11241.

11. Meyer LH, Karawajew L, Schrappe M, Ludwig WD, Debatin KM, Stahnke K. Cytochrome c-related caspase-3 activation determines treatment response and relapse in childhood precursor B-cell ALL. Blood 2006; 107: 4524-4531. 
12. Meyer LH, Queudeville M, Eckhoff SM, Creutzig U, Reinhardt D, Karawajew L et al. Intact apoptosis signaling in myeloid leukemia cells determines treatment outcome in childhood AML. Blood 2008; 111: 2899-2903.

13. Lock RB, Liem N, Farnsworth ML, Milross CG, Xue C, Tajbakhsh M et al. The nonobese diabetic/severe combined immunodeficient (NOD/SCID) mouse model of childhood acute lymphoblastic leukemia reveals intrinsic differences in biologic characteristics at diagnosis and relapse. Blood 2002; 99: 4100-4108.

14. Nijmeijer BA, Mollevanger P, van Zelderen-Bhola SL, Kluin-Nelemans HC, Willemze R, Falkenburg $\mathrm{JH}$. Monitoring of engraftment and progression of acute lymphoblastic leukemia in individual NOD/SCID mice. Exp Hematol 2001; 29: 322-329.

15. Notta F, Mullighan CG, Wang JC, Poeppl A, Doulatov S, Phillips LA et al. Evolution of human BCR-ABL1 lymphoblastic leukaemia-initiating cells. Nature 2011; 469: 362-367.

16. Schmitz M, Breithaupt P, Scheidegger N, Cario G, Bonapace L, Meissner B et al. Xenografts of highly resistant leukemia recapitulate the clonal composition of the leukemogenic compartment. Blood 2011; 118: 1854-1864.

17. Yeoh EJ, Ross ME, Shurtleff SA, Williams WK, Patel D, Mahfouz R et al. Classification, subtype discovery, and prediction of outcome in pediatric acute lymphoblastic leukemia by gene expression profiling. Cancer Cell 2002; 1: 133-143.

18. Armstrong SA, Staunton JE, Silverman LB, Pieters R, den Boer ML, Minden MD et al. $M L L$ translocations specify a distinct gene expression profile that distinguishes a unique leukemia. Nat Genet 2002; 30: 41-47.

19. Salomons GS, Smets LA, Verwijs-Janssen M, Hart AA, Haarman EG, Kaspers GJ et al. Bcl-2 family members in childhood acute lymphoblastic leukemia: relationships with features at presentation, in vitro and in vivo drug response and long-term clinical outcome. Leukemia 1999; 13: 1574-1580.

20. Wuchter C, Karawajew L, Ruppert V, Schrappe M, Harbott J, Ratei R et al. Constitutive expression levels of CD95 and Bcl-2 as well as CD95 function and spontaneous apoptosis in vitro do not predict the response to induction chemotherapy and relapse rate in childhood acute lymphoblastic leukaemia. Br J Haematol 2000; 110: 154-160.

21. Ong YL, McMullin MF, Bailie KE, Lappin TR, Jones FG, Irvine AE. High bax expression is a good prognostic indicator in acute myeloid leukaemia. Br J Haematol 2000; 111: 182-189.

22. Tamm I, Richter S, Oltersdorf D, Creutzig U, Harbott J, Scholz F et al. High expression levels of $x$-linked inhibitor of apoptosis protein and survivin correlate with poor overall survival in childhood de novo acute myeloid leukemia. Clin Cancer Res 2004; 10: 3737-3744

23. Schaich M, Illmer T, Seitz G, Mohr B, Schakel U, Beck JF et al. The prognostic value of Bcl$\mathrm{XL}$ gene expression for remission induction is influenced by cytogenetics in adult acute myeloid leukemia. Haematologica 2001; 86: 470-477.

24. Choi J, Hwang YK, Sung KW, Lee SH, Yoo KH, Jung HL et al. Expression of Livin, an antiapoptotic protein, is an independent favorable prognostic factor in childhood acute lymphoblastic leukemia. Blood 2007; 109: 471-477.

25. Carter BZ, Kornblau SM, Tsao T, Wang RY, Schober WD, Milella M et al. Caspaseindependent cell death in AML: caspase inhibition in vitro with pan-caspase inhibitors or in vivo by XIAP or Survivin does not affect cell survival or prognosis. Blood 2003; 102: $4179-4186$.

26. Tamm I, Kornblau SM, Segall H, Krajewski S, Welsh K, Kitada S et al. Expression and prognostic significance of IAP-family genes in human cancers and myeloid leukemias. Clin Cancer Res 2000; 6: 1796-1803.
27. Tamm I, Richter S, Scholz F, Schmelz K, Oltersdorf D, Karawajew L et al. XIAP expression correlates with monocytic differentiation in adult de novo AML: impact on prognosis. Hematol J 2004; 5: 489-495.

28. Kohler T, Schill C, Deininger MW, Krahl R, Borchert S, Hasenclever D et al. High Bad and Bax mRNA expression correlate with negative outcome in acute myeloid leukemia (AML). Leukemia 2002; 16: 22-29.

29. Faderl S, Thall P, Kantarjian H, Talpaz M, Harris D, Van Q et al. Caspase 2 and caspase 3 as predictors of complete remission and survival in adults with acute lymphoblastic leukemia. Clin Cancer Res 1999; 5: 4041-4007.

30. Cohen O, Inbal B, Kissil JL, Raveh T, Berissi H, Spivak-Kroizaman T et al. DAP-kinase participates in TNF-alpha- and Fas-induced apoptosis and its function requires the death domain. J Cell Biol 1999; 146: 141-148.

31. Inbal B, Cohen O, Polak-Charcon S, Kopolovic J, Vadai E, Eisenbach L et al. DAP kinase links the control of apoptosis to metastasis. Nature 1997; 390: 180-184.

32. Holleman A, den Boer ML, de Menezes RX, Cheok MH, Cheng C, Kazemier KM et al. The expression of 70 apoptosis genes in relation to lineage, genetic subtype, cellular drug resistance, and outcome in childhood acute lymphoblastic leukemia. Blood 2006; 107: 769-776.

33. Meyer LH, Debatin KM. Diversity of human leukemia xenograft mouse models: implications for disease biology. Cancer Res 2011; 71: 7141-7144.

34. Baersch G, Möllers T, Hötte A, Dockhorn-Dworniczak B, Rübe C, Ritter J et al. Good engraftment of B-cell precursor ALL in NOD-SCID mice. Klin Padiatr 1997; 209: 178-185.

35. Borgmann A, Baldy C, von Stackelberg A, Beyermann B, Fichtner I, Nurnberg P et al. Childhood ALL blasts retain phenotypic and genotypic characteristics upon long-term serial passage in NOD/SCID mice. Pediatr Hematol Oncol 2000; 17: 635-650.

36. Kamel-Reid S, Letarte M, Sirard C, Doedens M, Grunberger T, Fulop G et al. A model of human acute lymphoblastic leukemia in immune-deficient SCID mice. Science 1989; 246: 1597-1600.

37. Stahnke K, Mohr A, Liu J, Meyer LH, Karawajew L, Debatin KM. Identification of deficient mitochondrial signaling in apoptosis resistant leukemia cells by flow cytometric analysis of intracellular cytochrome c, caspase-3 and apoptosis. Apoptosis 2004; 9: $457-465$.

38. Manabe A, Coustan-Smith E, Behm FG, Raimondi SC, Campana D. Bone marrow-derived stromal cells prevent apoptotic cell death in B-lineage acute lymphoblastic leukemia. Blood 1992; 79: 2370-2377.

39. Campana D, Iwamoto S, Bendall L, Bradstock K. Growth requirements and immunophenotype of acute lymphoblastic leukemia progenitors. Blood 2005; 105: 4150.

40. Abramoff MD, Magalhaes PJ, Ram SJ. Image Processing with ImageJ. Biophotonics International 2004; 11: 36-42.

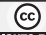

OMERIAHISRESERV

Cell Death and Disease is an open-access journal published by Nature Publishing Group. This work is licensed under the Creative Commons Attribution-NonCommercial-No Derivative Works 3.0 Unported License. To view a copy of this license, visit http://creativecommons.org/licenses/by-nc-nd/3.0/ 